\title{
Pediatric non-alcoholic fatty liver disease: an increasing public health issue
}

\author{
S. Berardis • E. Sokal
}

Received: 14 May 2013 / Accepted: 10 September 2013 /Published online: 26 September 2013

(C) The Author(s) 2013. This article is published with open access at Springerlink.com

\begin{abstract}
Non-alcoholic fatty liver disease (NAFLD) is a multifactorial condition that encompasses a wide spectrum of liver abnormalities ranging from simple liver steatosis to steatohepatitis (non-alcoholic steatohepatitis), which may be associated with fibrosis and progress to cirrhosis and endstage liver disease. NAFLD has recently become the most common cause of chronic liver disease in children and adolescents. NAFLD prevalence, alongside obesity, continues to increase among pediatric patients. Obesity is believed to represent a major risk factor for NAFLD, which is considered to be the liver presentation of the metabolic syndrome. Although the pathogenesis of NAFLD is not fully understood, the notion that multiple factors affect disease development and progression is widely accepted. Both genetic background and environmental factors contribute to NAFLD development. A more complete understanding of the pathogenesis may aid in developing non-invasive diagnostic tools and identifying new therapeutic targets. Liver biopsy currently remains the gold standard for NAFLD diagnosis and staging. Although lifestyle and diet modifications are key in NAFLD treatment, the development of new pharmacological therapies is crucial for patients who are unresponsive to first-line therapy. Conclusion: Pediatric NAFLD is an increasing public health issue that remains underdiagnosed. A large-scale screening in the high-risk population, especially among the overweight pediatric patients, should be considered, including measurement of serum transaminases and liver ultrasound. It is crucial to treat this condition as soon as possible in order to avoid the progression to end-stage liver disease.
\end{abstract}

S. Berardis $(\bowtie) \cdot$ E. Sokal

Service de gastroentérologie et hépatologie pédiatrique, Cliniques Universitaires Saint-Luc, Université catholique de Louvain, Avenue Hippocrate 10, 1200 Brussels, Belgium

e-mail: silvia.berardis@uclouvain.be
Keywords Liver fibrosis · Non-alcoholic fatty liver disease Pediatric patients $\cdot$ Steatohepatitis

\section{Introduction}

Over the past few decades, non-alcoholic fatty liver disease (NAFLD) has become the most common cause of chronic liver disease among children and adolescents in Western countries, with cases documented in children as young as 3 years of age [15]. NAFLD refers to a wide spectrum of liver abnormalities ranging from simple liver steatosis (fat accumulation in the liver) to steatohepatitis (non-alcoholic steatohepatitis, NASH), which may be associated with fibrosis and progress to cirrhosis and end-stage liver disease.

In 1952, S. Zelman documented liver lesions compatible with NASH for the first time [73]. Ludwig et al. used the term "NASH" in 1980 [32]. Three years later, the first pediatric NASH cases were reported [38].

NAFLD prevalence is increasing, alongside obesity, essentially because of sedentary lifestyles and hypercaloric diets [4]. NAFLD is considered the hepatic manifestation of the metabolic syndrome, which is characterized by insulin resistance, visceral obesity, hypertension, dyslipidemia, and abnormalities of fasting serum glucose levels. Pediatric patients with NAFLD have been shown to exhibit higher levels of insulin, total cholesterol, triglycerides, low-density lipoprotein cholesterol, and fasting glucose, as well as higher blood pressure values than obese children without NAFLD. Moreover, children with metabolic syndrome are more likely to display NAFLD than obese children without metabolic syndrome [61]. NAFLD is also associated with an increased risk of developing cardiovascular diseases [52]. NAFLD patients have a higher prevalence of atherosclerosis [50].

NAFLD pathogenesis is not yet fully understood, but the hypothesis that multiple factors contribute to disease development and progression is now widely accepted [3]. 
Histological liver analysis remains the gold standard to differentiate between simple steatosis and NASH, to perform disease staging, and to exclude other causes of chronic liver diseases[66].

No effective treatment is currently available. In children, weight loss and physical activity are considered first-line treatment. However, a poor compliance to lifestyle modifications is a daily reality. Developing new treatments to stop the disease from progressing to end-stage liver disease appears therefore crucial. Over the past several years, new pharmacological treatments have been developed and tested [5].

In this review, we will present an overview of current knowledge regarding the pathogenesis, diagnostic methods, histological features, and treatment of pediatric NAFLD.

\section{Epidemiology}

According to epidemiological data, NAFLD may affect 3$10 \%$ of pediatric patients, with a male-to-female ratio of $2: 1$

[4]. Obesity is thought to be the main risk factor for pediatric NAFLD. In obese children, NAFLD prevalence may reach $70-80 \%[36]$.

However, true NAFLD prevalence in children is unknown. Indeed, only a few studies have been conducted with children and adolescents. Moreover, in most studies on NAFLD prevalence in children, indirect measures such as blood tests and ultrasound were used as screening tools. Serum alanine aminotransferase (ALT) sensitivity and specificity are poor (64 and $81 \%$, respectively). Sensitivity is low, as some NAFLD children may present ALT values in the normal range [33]. Furthermore, various cutoff levels are cited in the literature. Ultrasound exhibits limited detection capabilities: this tool identifies only high levels of fat $(>30 \%)$ and cannot detect fibrosis [56]. Liver histology, the most relevant diagnosis criterion, is rarely used for screening [71].

More accurate estimates of disease prevalence in the USA were obtained from an autopsy study in San Diego. Steatosis was found in $9.6 \%$ of individuals aged 2 to 19 years and in $38 \%$ of obese children autopsied between 1993 and 2003 [59]. A liver biopsy study during gastric bypass surgery was performed in morbidly obese adolescents. This cohort showed a high NAFLD prevalence ( $83 \%$ ), with $20 \%$ of participants meeting the histological criteria for NASH [72].

NAFLD prevalence appears to increase with age, with a mean age at diagnosis between 11 and 13 years [71]. This tendency is likely explained by adolescent hormonal changes, which result in an increase in serum insulin levels and fat accumulation in the liver [55]. Ethnicity may also affect NAFLD prevalence. Hispanic children have the highest NAFLD prevalence (36\%), whereas African American children are less affected (14\%), although both populations exhibit similar obesity rates [21]. These discrepancies may be accounted for by genetic factors or environmental features, such as diet and exercise [16].

The prognosis of pediatric NAFLD is still unknown, as only a limited number of long-term follow-up studies have been conducted to date [3]. The first long-term study with a follow-up period of up to 20 years, which assessed overall survival of NAFLD pediatric patients, reported a shorter longterm survival than non-affected patients [71].

\section{Pathogenesis}

NAFLD was initially considered a two-hit process. At present, however, the notion that NAFLD pathogenesis is multifactorial with many factors affecting disease development and progression is widely accepted. The "multiple-hit" hypothesis is currently the established pathogenetic model [17].

At disease onset, NAFLD is characterized by fat accumulation in the liver (steatosis) and insulin resistance, influenced by genetic susceptibility, epigenetic mechanisms, a sedentary lifestyle, and hypercaloric diets [48]. Hepatic triglyceride accumulation results from increased delivery of free fatty acids to the liver, increased lipogenesis, and impaired fatty acid metabolism in hepatocytes. Hepatic fat accumulation has been shown to exacerbate insulin resistance by interfering with phosphorylation of insulin receptor substrates [57]. Free fatty acid accumulation and insulin resistance may predispose the fatty liver to secondary hits, including oxidative stress, mitochondrial dysfunction, pro-inflammatory cytokines imbalance, and stellate cells activation, which lead to necroinflammation and fibrosis [64] (Fig. 1).

The gut's critical role in NAFLD pathogenesis has recently been given consideration. In NAFLD, an alteration of gut microbiota and enhanced gut permeability increase liver exposure to gut-derived bacterial products, such as lipopolysaccharides. These products stimulate innate immune receptors (Toll-like receptors), which leads to activation of the signalling pathways involved in liver inflammation and fibrogenesis [22].

Hepatic stellate cells are considered the main extracellular matrix-producing cells during NASH development. However, the hepatic progenitor cell compartment of the liver has recently been shown to be expanded in children with NAFLD. Hepatic progenitor cell activation appears to play a role in liver response to oxidative stress and is correlated with fibrosis and NASH progression [43].

Adipocytokines, including adiponectin, leptin, resistin, and tumour necrosis factor-alpha (TNF-alpha), also appear to be involved in the progression of simple steatosis to NASH. Adipocytes or inflammatory cells infiltrating the adipose tissue in insulin resistance conditions are responsible for adipocytokine secretion. Leptin may activate hepatic stellate cells and suppress their apoptosis. 
Fig. 1 Pathogenesis of NAFLD: the "multiple-hit" hypothesis.

Genetics, epigenetics, environmental factors, insulin resistance, and obesity influence fat accumulation in the liver (steatosis). The fatty liver is then predisposed to secondary hits, including oxidative stress, mitochondrial dysfunction, proinflammatory cytokines imbalance, and stellate cells activation, which lead to necroinflammation and fibrosis ( $N A S H$ non-alcoholic steatohepatitis)
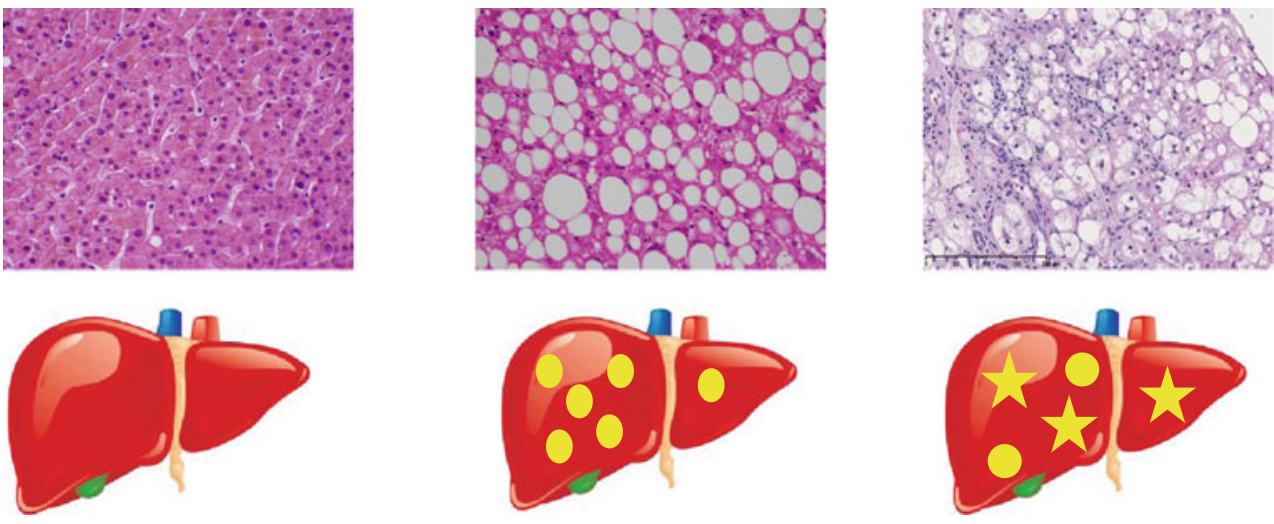

Normal liver

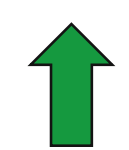

NAFLD

NASH

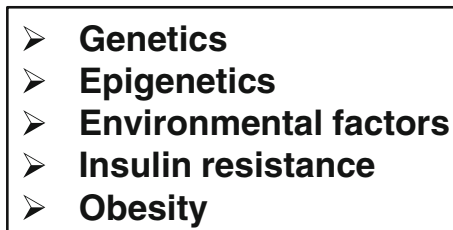

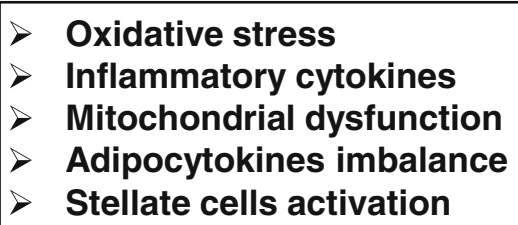

The expansion of adipose tissue, and particularly that of visceral fat, is associated with a decrease in the release of insulin-sensitizing and anti-inflammatory cytokines and an increase in the release of pro-inflammatory molecules [34]. TNF-alpha and IL-6 levels are often elevated in the liver and blood of NASH patients. These cytokines are involved in Kupffer cell recruitment and activation, as well as in hepatic stellate cell activation in myofibroblasts [48].

To summarize, NAFLD results from crosstalk between multiple organs, including adipose tissue, the pancreas, gut, and liver.

\section{Diagnosis of NAFLD/NASH}

NAFLD is often diagnosed in asymptomatic patients, with unexplained increased serum aminotransferase or gammaglutamyl transpeptidase values detected during routine checkups. However, some patients may suffer from abdominal pain; hepatomegaly may be present, whereas splenomegaly is rare.

For diagnosing NAFLD, it is necessary to eliminate other liver disease etiologies, such as hepatitis B and C, autoimmune hepatitis, drug-induced liver injury, Wilson's disease, alpha 1-antitrypsin deficiency, inborn errors of fatty acid or carnitine metabolism, peroxisomal disorders, lysosomal storage disorders, and cystic fibrosis. However, positive serum autoantibodies (antinuclear and anti-SMA) are often present in NAFLD pediatric patients, in the absence of autoimmune hepatitis. Their clinical significance remains unclear [3].
Indirect markers

Enhanced ALT levels are common among pediatric patients with NAFLD [51]. Aminotransferase levels may range from normal to four to six times the upper limit of normal. Mild aminotransferase elevation is usually observed in NAFLD patients (1.5-2 times the upper limit of normal) [8]. However, circulating aminotransferases levels are frequently normal in children with NAFLD and NASH. Furthermore, normal aminotransferase levels do not exclude possible fibrosis or cirrhosis. Together with fibrosis progression and steatosis reduction, aminotransferase levels may decrease. Therefore, this test is not representative of NAFLD severity. Moreover, dietary habits and hyperalimentation may impact on serum aminotransferase levels [24].

Lipid profiles, fasting glucose, and insulin levels should be evaluated in children with NAFLD, who often present with several metabolic syndrome components [3].

\section{Imaging techniques}

Ultrasonography (US) is the most common imaging modality for fatty liver detection. US has several advantages, such as its relatively low cost and wide availability. A recent study demonstrated liver US efficacy for quantifying steatosis in children. A strong correlation between US steatosis scores and steatosis severity on liver biopsy was observed [62]. However, US sensitivity decreases when the liver contains $<30 \%$ fat or 
in individuals with $\mathrm{BMI} \geq 40$ [56]. Moreover, US cannot exclude steatohepatitis or fibrosis.

Computed tomography is a more sensitive technique for detecting fat in the liver, but its use is not recommended in children because of radiation exposure. Magnetic resonance imaging is also relatively sensitive in liver fat quantification, although its costs and the need for sedation in young children limit its clinical utility. Finally, none of these imaging tools exhibits sufficient sensitivity and specificity to differentiate between simple steatosis and NASH or to perform disease staging [53].

Transient elastography is an accurate tool for liver fibrosis detection and has been validated for liver fibrosis assessment in several liver diseases. A study conducted in children and adolescents with NAFLD showed that transient elastography is an accurate and reproducible methodology for identifying children without fibrosis or with advanced fibrosis [49]. However, this tool is unable to discriminate between intermediate degrees of fibrosis and thus unsuited for providing reliable disease stage indications [47].

\section{Liver biopsy}

Liver biopsy remains the gold standard in NAFLD diagnosis. Indeed, this is the only way to distinguish between simple steatosis and NASH, to determine the severity of liver damage and inflammatory activity, and to assess the degree of fibrosis. Yet liver biopsy is an invasive method that may be associated with complication, such as bleeding. Therefore, research has been undertaken to develop non-invasive tools capable of monitoring disease evolution and therapeutic responses.

Histology remains also a useful tool to exclude other causes of chronic liver disease, as mentioned above.

Development of non-invasive tools

Certain clinical parameters, such as age, insulin resistance, BMI, waist circumference, and lipid and glucose profiles, may be useful for identifying children with potentially severe NAFLD [66]. The pediatric NAFLD fibrosis index (PNFI) was developed on the basis of three clinical parameters: age, waist circumference, and triglyceride levels. This index aims to predict liver fibrosis in children with NAFLD [41]. Although the index provides a good positive predictive value, its negative predictive value for ruling out fibrosis is not optimal.

Recently, the combination of the enhanced liver fibrosis test and PNFI has proved relatively accurate for predicting liver fibrosis in children with NAFLD [6]. The enhanced liver fibrosis test includes a panel of serum markers that are extracellular matrix components, namely the tissue inhibitor of metalloproteinase-1, hyaluronic acid, and amino-terminal propeptide of type III collagen.

Biomarkers related to hepatocyte apoptosis have been developed in order to stratify disease severity in pediatric NAFLD. Caspase-generated cytokeratin-18 fragments are specific by-products of liver cell apoptosis. Fitzpatrick et al. showed that children with NAFLD have elevated levels of cytokeratin-18 fragments, and that those with NASH have markedly higher levels of cytokeratin-18 fragments [20].

Several other biomarkers of oxidative stress, inflammation, and fibrosis are currently under investigation. However, larger studies must be undertaken before using these markers in pediatric clinics [19].

- NAFLD is often diagnosed in asymptomatic patients with unexplained increased ALT levels.

- The ALT level is not correlated with NAFLD severity.

- Ultrasonography can detect steatosis when the liver contains more than $30 \%$ fat but cannot exclude steatohepatitis or fibrosis.

- Liver biopsy remains the gold standard for the diagnosis of NAFLD.

\section{Histology}

Children with NAFLD exhibit the same morphological lesions as adults, namely hepatocellular injury, inflammation, and fibrosis. However, there is a unique pattern of lesion distribution in children. This pattern is characterized by macrovesicular hepatocellular steatosis, portal inflammation, and portal fibrosis in the absence of ballooning [26].

In 2005, Schwimmer et al. described two different forms of pediatric steatohepatitis. Type 1, which is characterized by the start of steatosis in the perivenular zone, ballooning, and perisinusoidal fibrosis, is similar to the adult pattern. Type 2 is characterized by periportal or panacinar steatosis, portal inflammation, and portal fibrosis in the absence of ballooning. This pattern was found in the majority of children [58]. This classification was not widely used as, a few years later, an overlap pattern combining features of both types was found in $>50 \%$ NAFLD children [14].

Potential mechanisms that explain the different histological patterns between adults and children remain unclear. However, a recent study by Swiderska-Syn et al. hypothesized that the Hedgehog pathway, which is involved in fibro-ductular response, may account for the unique histological features of pediatric NAFLD. In fact, the portal and periportal compartments of pre-pubescent male livers were shown to exhibit high Hedgehog pathway activity [63].

\section{Steatosis}

Steatosis refers to the abnormal accumulation of fat in hepatocytes. The minimum criterion for NAFLD diagnosis is the presence of steatosis in $>5 \%$ hepatocytes [64]. In NAFLD, steatosis is mainly macrovacuolar. On occasion, microvacuolar steatosis may be found [12]. In NAFLD, steatosis may also affect the whole parenchyma. 
The pattern of steatosis distribution is different in children than in adults. In adults, steatosis starts in the perivenular zone (zone 3), whereas in children, it commonly begins in the periportal zone [58].

Ballooning

Ballooning is the main morphological feature of hepatocellular damage from NASH. Ballooning is defined as a cellular enlargement of 1.5-2 times the normal hepatocyte diameter. The hepatocyte cytoplasm is clarified, rarefied, and may contain Mallory-Denk bodies, which are eosinophilic inclusions composed of cytoskeletal peptides aggregates. In adults, ballooning is usually observed in zone 3; in children, MalloryDenk bodies and ballooned hepatocytes are rather uncommon [3].

\section{Inflammation}

Inflammatory infiltrates, a key marker of NASH, are usually composed of lymphocytes, histiocytes, and Kupffer cells, with only few granulocytes found [9]. In adults, inflammation is prevalent in lobular spaces, whereas in children, infiltrates are found mainly in the portal tracts [2].

Fibrosis

Fibrosis, a histological marker of chronic liver damage, typically starts in acinar zone 3 in adults, with a characteristic chicken wire pattern. In children, fibrosis is observed mainly in the portal-periportal tract. In advanced disease stages, bridging fibrosis and cirrhosis may develop [11].

\section{Scoring systems}

Several scoring systems have been developed, of which two most widely used are the Brunt Score for NASH [10] and the NAFLD activity score, developed by the National Institute of Diabetes and Digestive and Kidney Diseases and the NASH Clinical Research Network for NAFLD [25], respectively. Both systems provide a numerical score that allows for grading disease activity and staging fibrosis.

Since the detection of histological pattern differences between NAFLD children and adults, a new grading score (PNHS) has been developed for pediatric NAFLD. This scoring system accounts for the presence of portal inflammation, with a weighing of each histological feature [7].

\section{Treatment}

As previously mentioned, NAFLD may progress to cirrhosis and end-stage liver disease. Therefore, patients affected by this condition should be treated as soon as possible.
Diet and lifestyle modifications

The first-line treatment in all NAFLD patients concerns lifestyle modifications so as to achieve gradual weight loss. By reducing dietary intake and increasing physical activity, patients may achieve weight loss. These lifestyle modifications may positively impact on serum aminotransferase levels and various metabolic parameters, such as insulin resistance, fasting glucose, and lipids [46, 54]. Improvement in insulin resistance appears to be the parameter most strongly associated with NAFLD improvement. Insulin resistance can be evaluated using the homeostasis model assessment of insulin resistance (HOMA-IR) [27].

Nobili et al. showed that liver histology significantly improved following lifestyle modifications over a 24-month period, with improvements in steatosis, inflammation, hepatocyte ballooning, and NAFLD activity score observed [45].

It should, however, be noted that children's diets must be well-balanced, with respect of the food pyramid principle, in order to promote harmonic growth. A follow-up by a dietician is therefore crucial.

Currently, no evidence-based guidelines that recommend a particular diet or exercise are available. However, a reduction in fast-release carbohydrate consumption, especially that of fructose, is advised not only to improve insulin resistance and reduce lipogenesis, but also to counteract the pro-inflammatory and fibrogenic effects of fructose [1].

Yet, compliance to such lifestyle modifications is often poor, especially in adolescents. A multidisciplinary approach and a support by a dietician may be helpful in order to assess diet quality and measure caloric intake.

Bariatric surgery has been shown to improve liver damage in adults [70]. However, very few data are available for adolescents. Standardization of eligibility criteria for adolescents and further studies on safety and long-term efficacy of this approach are warranted [48].

\section{Pharmacotherapy}

To avoid severe organ damage, pharmacological therapies must be developed for children who do not adhere to or are unresponsive to lifestyle modifications.

\section{Vitamin E}

In pediatric studies, vitamin $\mathrm{E}$ efficacy was shown not to be superior to lifestyle changes alone $[67,69]$. In a larger and more recent study (TONIC study), the effects of vitamin E, metformin, or placebo were assessed in NAFLD children. Although vitamin E treatment was not superior to placebo in terms of ALT level reduction, histological hepatocellular ballooning was shown to be improved under vitamin E treatment in children with biopsy-proven NASH [28]. 


\section{Metformin}

Metformin, an insulin-sensitizing agent, lowers hepatic glucose production and promotes glucose uptake in muscles. In 2005, Schwimmer et al. documented a hepatic steatosis reduction, evaluated using magnetic resonance spectroscopy and ALT levels, in non-diabetic children with biopsy-proven NASH who were treated with metformin $500 \mathrm{mg}$ twice daily for 24 weeks [60]. A 2009 study, involving a cohort of 50 obese, multi-ethnic, and insulin-resistant adolescents, showed improvements in fatty liver prevalence and severity and in fasting insulin levels in metformin-treated patients in comparison with placebo-treated patients [39]. However, in a pediatric study comparing metformin with lifestyle interventions, Nobili et al. were not able to confirm metformin's advantages in NAFLD [44]. More recently, the TONIC study demonstrated that neither metformin nor vitamin $\mathrm{E}$ was superior to placebo in reducing ALT levels, whereas both approaches improved hepatocellular ballooning, with no significant improvements observed among other histological features [28].

\section{Ursodeoxycholic acid}

Ursodeoxycholic acid (UDCA), a hepatoprotective agent, is assumed to prevent NAFLD progression by protecting hepatocytes from bile salt-mediated mitochondrial injury, activating anti-apoptotic signalling pathways, and fulfilling immunomodulatory functions.

The potential efficacy of UDCA was evaluated in a randomized controlled trial involving 31 NAFLD children. In this study, UDCA ( $10 \mathrm{mg} / \mathrm{kg} /$ day) was shown to be ineffective in treating liver abnormalities in obese children [65].

\section{Probiotics}

With growing evidence that gut microbiota contributes to NAFLD progression, probiotics have been considered for NAFLD treatment. Studies undertaken on NAFLD animal models suggested that probiotics may reduce liver inflammation and improve gut epithelial barrier function $[13,18]$. In 2005, Loguerico et al. evaluated the effect of a probiotic (VSL\#3) in patients with chronic hepatopathies, including NAFLD. In this study, probiotics were shown to reduce liver injury and improve liver function tests [31]. In 2011, a doubleblind, placebo-controlled pilot study was performed in obese children with persisting hypertransaminasemia and ultrasonographic bright liver. In this study, patients receiving probiotic therapy (Lactobacillus GG) exhibited a significant improvement in serum ALT and anti-peptidoglycan polysaccharide antibody levels that was independent of changes in BMI $z$ score and visceral fat [68]. Based on these study findings, probiotics may be considered a promising tool for pediatric NAFLD treatment.

\section{Polyunsaturated fatty acids}

Polyunsaturated fatty acids include essential fatty acids, such as omega-3 and omega-6. Recent studies, performed in NAFLD animal models and in adults, evaluated the effects of oral therapy using omega-3 fatty acids. These studies demonstrated fatty acids' anti-inflammatory and insulinsensitizing properties, suggesting their potential role in NAFLD treatment [35]. In a recent double-blind, randomized, controlled trial conducted in children with NAFLD, a 6-month treatment with omega-3-docosahexaenoic acid (DHA) was reported to improve liver steatosis and insulin sensitivity, with no significant differences observed between the 250 and $500 \mathrm{mg} /$ day doses [42]. These positive results were confirmed after a 24-month treatment period. Moreover, in the DHAtreated groups, triglyceride levels were lower after a 24-month treatment period, with lower ALT levels observed after a 12month treatment period [40].

Novel therapeutic targets

Pentoxifylline, a phosphodiesterase inhibitor, antagonizes the TNF-alpha pathway. In NASH adults, this treatment was shown to promote a reduction in serum ALT levels and an improvement in histological features [30].

Farnesoid X receptors (FXR), which are expressed in the bowel and liver, are likely involved in NAFLD pathogenesis by mediating control of lipid and glucose homeostasis and bacterial flora growth. A reduction in hepatic inflammation and fibrogenesis may be induced through various mechanisms, and FXR agonists may have a potential role in NAFLD treatment. However, further studies must be undertaken before this agent can be considered a valid NAFLD treatment [23].

Toll-like receptors (TLR) are implicated in the pathogenesis of NAFLD. TLR stimulation results in inflammatory response activation, which plays a role in the progression of NAFLD to NASH. Therefore, TLR antagonists may prove to be potential therapeutic targets for pediatric NAFLD. However, further studies are required to support this hypothesis [37].

Incretin mimetics and dipeptidyl peptidase-4 (DPP-4) inhibitors increase insulin secretion. Glucagon-like peptide-1 (GLP-1), which is an incretin secreted in response to food intake, stimulates insulin secretion and inhibits glucagon release. DPP-4 is an enzyme implicated in the degradation of circulating GLP-1. Studies conducted in animals and in human adults demonstrated the efficacy of GLP-1 receptor agonists, which were resistant to DPP-4 degradation or DPP-4 inhibitors. These pharmacological agents represent potentially new therapeutic approaches to NAFLD treatment by 


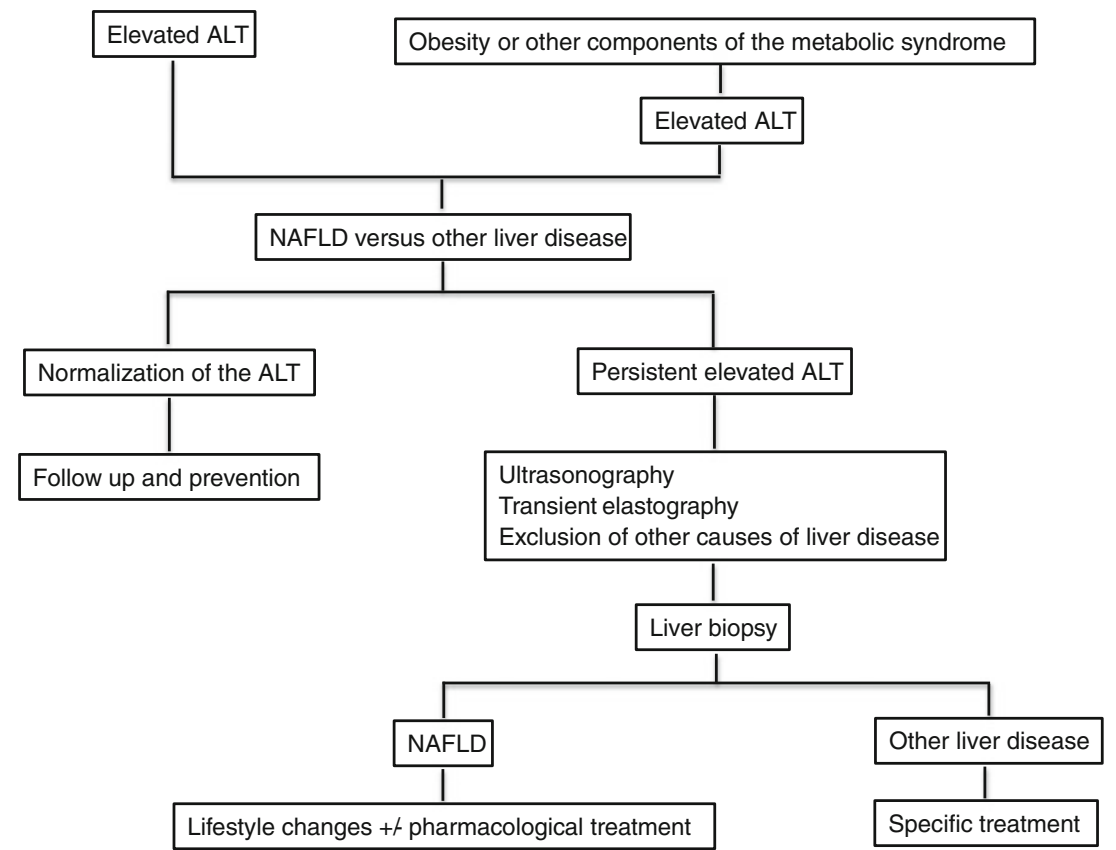

Fig. 2 Clinical algorithm for the management of pediatric NAFLD. In case of unexplained elevated ALT, of obesity, and/or of other comorbidities (insulin resistance, dyslipidemia, diabetes mellitus, hypertension), we recommend to perform a dosage of the liver transaminases. In case of normalization of the liver tests, the child has to be followed regularly by his pediatrician and a prevention should be introduced (diet and exercise). If the ALT remain elevated, an ultrasonography (and if necessary, a transient elastography) is recommended in order to detect the

increasing fatty acid oxidation, decreasing lipogenesis, and improving hepatic glucose metabolism [29].

Nowadays, there is no real consensus on the treatments for pediatric NAFLD. The benefits of the lifestyle modifications have widely been accepted. However, some controversies persist concerning the pharmacological treatments. The best way to manage this condition is may be to adapt the treatment to each patient, considering also existing co-morbidities (such as insulin resistance or diabetes mellitus for which metformin can be useful). Vitamin E, probiotics, and polyunsaturated fatty acids emerge as potentially useful treatments, although appropriate controlled studies remain necessary before their universal recommendation.

We propose an algorithm for the management of pediatric NAFLD, in order to help pediatricians facing the problem of this condition (Fig. 2).

- Lifestyle modifications represent the first-line treatment of NAFLD.

They can have a positive impact on ALT levels and can improve liver histology.

- A reduction in fast-release carbohydrates consumption (especially fructose) is advised.

- A multidisciplinary approach is crucial.

- Pharmacological treatments should be considered for patients who do not adhere or are unresponsive to lifestyle modifications. presence of steatosis. Other causes of liver diseases should also be excluded. If NAFLD is suspected or if the etiology of the liver disease remains unclear, a liver biopsy is recommended in order to detect the presence of inflammation and/or fibrosis, to stage the disease, and to confirm the diagnosis. If the diagnosis of NAFLD is confirmed, lifestyle changes represent the first-line treatment. Pharmacological treatments can be considered for patients who do not adhere to or are unresponsive to lifestyle modifications

\section{Conclusion}

While pediatric NAFLD is an increasing public health issue, this condition is still underdiagnosed, as NAFLD pediatric patients are often asymptomatic. Over the course of the disease, NAFLD may progress to NASH and end-stage liver disease. Therefore, it is crucial to diagnose and treat this condition at an early stage, and high-risk population screening should be performed. Liver biopsy remains the gold standard for NAFLD diagnosis and staging. Although some non-invasive tools have recently been developed for monitoring disease evolution and therapeutic response, there is an urgent need for new tools that are suitable for large-scale pediatric NAFLD screening.

First-line therapy focuses on diet and lifestyle modifications, and to this end, a multidisciplinary approach is recommended. However, in the case of poor compliance or in the absence of response to lifestyle changes, pharmacological therapies should be initiated in order to prevent severe organ damage. Further basic research studies are required in order to identify new therapeutic targets.

Finally, the role of the pediatrician is also to detect the overweight among their patients and to give them advices in order to prevent obesity and subsequently NAFLD. 
Open Access This article is distributed under the terms of the Creative Commons Attribution License which permits any use, distribution, and reproduction in any medium, provided the original author(s) and the source are credited.

\section{References}

1. Abdelmalek MF, Suzuki A, Guy C, Unalp-Arida A, Colvin R, Johnson RJ, Diehl AM (2010) Increased fructose consumption is associated with fibrosis severity in patients with nonalcoholic fatty liver disease. Hepatology 51:1961-1971

2. Alisi A, Bedogni G, De VR, Comparcola D, Manco M, Nobili V (2011) Relationship between portal chronic inflammation and disease severity in paediatric non-alcoholic fatty liver disease. Dig Liver Dis 43:143-146

3. Alisi A, Feldstein AE, Villani A, Raponi M, Nobili V (2012) Pediatric nonalcoholic fatty liver disease: a multidisciplinary approach. Nat Rev Gastroenterol Hepatol 9:152-161

4. Alisi A, Manco M, Vania A, Nobili V (2009) Pediatric nonalcoholic fatty liver disease in 2009. J Pediatr 155:469-474

5. Alisi A, Nobili V (2011) Nonalcoholic fatty liver disease: targeted therapy in children - what is the right way? Nat Rev Gastroenterol Hepatol 8:425-426

6. Alkhouri N, Carter-Kent C, Lopez R, Rosenberg WM, Pinzani M, Bedogni G, Feldstein AE, Nobili V (2011) A combination of the pediatric NAFLD fibrosis index and enhanced liver fibrosis test identifies children with fibrosis. Clin Gastroenterol Hepatol 9:150-155

7. Alkhouri N, De VR, Alisi A, Yerian L, Lopez R, Feldstein AE, Nobili V (2012) Development and validation of a new histological score for pediatric non-alcoholic fatty liver disease. J Hepatol 57:1312-1318

8. Angulo P (2002) Nonalcoholic fatty liver disease. N Engl J Med 346: 1221-1231

9. Brunt EM (2010) Pathology of nonalcoholic fatty liver disease. Nat Rev Gastroenterol Hepatol 7:195-203

10. Brunt EM, Janney CG, Di Bisceglie AM, Neuschwander-Tetri BA, Bacon BR (1999) Nonalcoholic steatohepatitis: a proposal for grading and staging the histological lesions. Am J Gastroenterol 94:24672474

11. Brunt EM, Tiniakos DG (2010) Histopathology of nonalcoholic fatty liver disease. World J Gastroenterol 16:5286-5296

12. Burt AD, Mutton A, Day CP (1998) Diagnosis and interpretation of steatosis and steatohepatitis. Semin Diagn Pathol 15:246-258

13. Cani PD, Bibiloni R, Knauf C, Waget A, Neyrinck AM, Delzenne NM, Burcelin R (2008) Changes in gut microbiota control metabolic endotoxemia-induced inflammation in high-fat diet-induced obesity and diabetes in mice. Diabetes 57:1470-1481

14. Carter-Kent C, Yerian LM, Brunt EM, Angulo P, Kohli R, Ling SC, Xanthakos SA, Whitington PF, Charatcharoenwitthaya P, Yap J, Lopez R, McCullough AJ, Feldstein AE (2009) Nonalcoholic steatohepatitis in children: a multicenter clinicopathological study. Hepatology 50:1113-1120

15. Day CP (2011) Non-alcoholic fatty liver disease: a massive problem. Clin Med 11:176-178

16. Deboer MD (2011) Ethnicity, obesity and the metabolic syndrome: implications on assessing risk and targeting intervention. Expert Rev Endocrinol Metab 6:279-289

17. Della CC, Alisi A, Saccari A, De VR, Vania A, Nobili V (2012) Nonalcoholic fatty liver in children and adolescents: an overview. J Adolesc Health 51:305-312

18. Esposito E, Iacono A, Bianco G, Autore G, Cuzzocrea S, Vajro P, Canani RB, Calignano A, Raso GM, Meli R (2009) Probiotics reduce the inflammatory response induced by a high-fat diet in the liver of young rats. J Nutr 139:905-911
19. Feldstein AE, Nobili V (2010) Biomarkers in nonalcoholic fatty liver disease: a new era in diagnosis and staging of disease in children. $\mathrm{J}$ Pediatr Gastroenterol Nutr 51:378-379

20. Fitzpatrick E, Mitry RR, Quaglia A, Hussain MJ, DeBruyne R, Dhawan A (2010) Serum levels of CK18 M30 and leptin are useful predictors of steatohepatitis and fibrosis in paediatric NAFLD. J Pediatr Gastroenterol Nutr 51:500-506

21. Fraser A, Longnecker MP, Lawlor DA (2007) Prevalence of elevated alanine aminotransferase among US adolescents and associated factors: NHANES 1999-2004. Gastroenterology 133:1814-1820

22. Frasinariu OE, Ceccarelli S, Alisi A, Moraru E, Nobili V (2012) Gutliver axis and fibrosis in nonalcoholic fatty liver disease: an input for novel therapies. Dig Liver Dis 45(7):543-551

23. Fuchs M (2012) Non-alcoholic Fatty liver disease: the bile Acidactivated farnesoid $\mathrm{X}$ receptor as an emerging treatment target. $\mathrm{J}$ Lipids 2012:934396

24. Kechagias S, Ernersson A, Dahlqvist O, Lundberg P, Lindstrom T, Nystrom FH (2008) Fast-food-based hyper-alimentation can induce rapid and profound elevation of serum alanine aminotransferase in healthy subjects. Gut 57:649-654

25. Kleiner DE, Brunt EM, Van NM, Behling C, Contos MJ, Cummings OW, Ferrell LD, Liu YC, Torbenson MS, Unalp-Arida A, Yeh M, McCullough AJ, Sanyal AJ (2005) Design and validation of a histological scoring system for nonalcoholic fatty liver disease. Hepatology 41:1313-1321

26. Ko JS, Yoon JM, Yang HR, Myung JK, Kim H, Kang GH, Cheon JE, Seo JK (2009) Clinical and histological features of nonalcoholic fatty liver disease in children. Dig Dis Sci 54:2225-2230

27. Koot BG, van der Baan-Slootweg OH, Tamminga-Smeulders CL, Rijcken TH, Korevaar JC, van Aalderen WM, Jansen PL, Benninga MA (2011) Lifestyle intervention for non-alcoholic fatty liver disease: prospective cohort study of its efficacy and factors related to improvement. Arch Dis Child 96:669-674

28. Lavine JE, Schwimmer JB, Van Natta ML, Molleston JP, Murray KF, Rosenthal P, Abrams SH, Scheimann AO, Sanyal AJ, Chalasani N, Tonascia J, Unalp A, Clark JM, Brunt EM, Kleiner DE, Hoofnagle JH, Robuck PR (2011) Effect of vitamin E or metformin for treatment of nonalcoholic fatty liver disease in children and adolescents: the TONIC randomized controlled trial. JAMA 305:1659-1668

29. Lee J, Hong SW, Rhee EJ, Lee WY (2012) GLP-1 receptor agonist and non-alcoholic fatty liver disease. Diabetes Metab J 36:262-267

30. Li W, Zheng L, Sheng C, Cheng X, Qing L, Qu S (2011) Systematic review on the treatment of pentoxifylline in patients with nonalcoholic fatty liver disease. Lipids Health Dis 10:49

31. Loguercio C, Federico A, Tuccillo C, Terracciano F, D'Auria MV, De SC, Del Vecchio BC (2005) Beneficial effects of a probiotic VSL\#3 on parameters of liver dysfunction in chronic liver diseases. J Clin Gastroenterol 39:540-543

32. Ludwig J, Viggiano TR, McGill DB, Oh BJ (1980) Nonalcoholic steatohepatitis: Mayo Clinic experiences with a hitherto unnamed disease. Mayo Clin Proc 55:434-438

33. Manco M, Alisi A, Nobili V (2008) Risk of severe liver disease in NAFLD with normal ALT levels: a pediatric report. Hepatology 48: 2087-2088

34. Marra F, Gastaldelli A, Svegliati BG, Tell G, Tiribelli C (2008) Molecular basis and mechanisms of progression of non-alcoholic steatohepatitis. Trends Mol Med 14:72-81

35. Masterton GS, Plevris JN, Hayes PC (2010) Review article: omega-3 fatty acids - a promising novel therapy for non-alcoholic fatty liver disease. Aliment Pharmacol Ther 31:679-692

36. Mencin AA, Lavine JE (2011) Nonalcoholic fatty liver disease in children. Curr Opin Clin Nutr Metab Care 14:151-157

37. Miura K, Seki E, Ohnishi H, Brenner DA (2010) Role of toll-like receptors and their downstream molecules in the development of nonalcoholic fatty liver disease. Gastroenterol Res Pract 2010: 362847 
38. Moran JR, Ghishan FK, Halter SA, Greene HL (1983) Steatohepatitis in obese children: a cause of chronic liver dysfunction. Am J Gastroenterol 78:374-377

39. Nadeau KJ, Ehlers LB, Zeitler PS, Love-Osborne K (2009) Treatment of non-alcoholic fatty liver disease with metformin versus lifestyle intervention in insulin-resistant adolescents. Pediatr Diabetes 10:5-13

40. Nobili V, Alisi A, Della CC, Rise P, Galli C, Agostoni C, Bedogni G (2012) Docosahexaenoic acid for the treatment of fatty liver: randomised controlled trial in children. Nutr Metab Cardiovasc Dis. doi:10.1016/j.numecd.2012.10.010

41. Nobili V, Alisi A, Vania A, Tiribelli C, Pietrobattista A, Bedogni G (2009) The pediatric NAFLD fibrosis index: a predictor of liver fibrosis in children with non-alcoholic fatty liver disease. BMC Med 7:21

42. Nobili V, Bedogni G, Alisi A, Pietrobattista A, Rise P, Galli C, Agostoni C (2011) Docosahexaenoic acid supplementation decreases liver fat content in children with non-alcoholic fatty liver disease: double-blind randomised controlled clinical trial. Arch Dis Child 96: 350-353

43. Nobili V, Carpino G, Alisi A, Franchitto A, Alpini G, De VR, Onori P, Alvaro D, Gaudio E (2012) Hepatic progenitor cells activation, fibrosis, and adipokines production in pediatric nonalcoholic fatty liver disease. Hepatology 56:2142-2153

44. Nobili V, Manco M, Ciampalini P, Alisi A, Devito R, Bugianesi E, Marcellini M, Marchesini G (2008) Metformin use in children with nonalcoholic fatty liver disease: an open-label, 24-month, observational pilot study. Clin Ther 30:1168-1176

45. Nobili V, Manco M, Devito R, Di C, V, Comparcola D, Sartorelli MR, Piemonte F, Marcellini M, Angulo P (2008) Lifestyle intervention and antioxidant therapy in children with nonalcoholic fatty liver disease: a randomized, controlled trial. Hepatology 48:119-128

46. Nobili V, Marcellini M, Devito R, Ciampalini P, Piemonte F, Comparcola D, Sartorelli MR, Angulo P (2006) NAFLD in children: a prospective clinical-pathological study and effect of lifestyle advice. Hepatology 44:458-465

47. Nobili V, Monti L, Alisi A, Lo ZC, Pietrobattista A, Toma P (2011) Transient elastography for assessment of fibrosis in paediatric liver disease. Pediatr Radiol 41:1232-1238

48. Nobili V, Svegliati-Baroni G, Alisi A, Miele L, Valenti L, Vajro P (2012) A 360-degree overview of paediatric NAFLD: recent insights. J Hepatol 58(6):1218-1229

49. Nobili V, Vizzutti F, Arena U, Abraldes JG, Marra F, Pietrobattista A, Fruhwirth R, Marcellini M, Pinzani M (2008) Accuracy and reproducibility of transient elastography for the diagnosis of fibrosis in pediatric nonalcoholic steatohepatitis. Hepatology 48:442-448

50. Pacifico L, Nobili V, Anania C, Verdecchia P, Chiesa C (2011) Pediatric nonalcoholic fatty liver disease, metabolic syndrome and cardiovascular risk. World J Gastroenterol 17:3082-3091

51. Patel DA, Srinivasan SR, Chen W, Berenson GS (2011) Serum alanine aminotransferase and its association with metabolic syndrome in children: the bogalusa heart study. Metab Syndr Relat Disord 9:211-216

52. Perseghin G (2010) The role of non-alcoholic fatty liver disease in cardiovascular disease. Dig Dis 28:210-213

53. Qayyum A, Chen DM, Breiman RS, Westphalen AC, Yeh BM, Jones KD, Lu Y, Coakley FV, Callen PW (2009) Evaluation of diffuse liver steatosis by ultrasound, computed tomography, and magnetic resonance imaging: which modality is best? Clin Imaging 33:110-115

54. Reinehr T, Schmidt C, Toschke AM, Andler W (2009) Lifestyle intervention in obese children with non-alcoholic fatty liver disease: 2-year follow-up study. Arch Dis Child 94:437-442
55. Roberts EA (2007) Pediatric nonalcoholic fatty liver disease (NAFLD): a "growing" problem? J Hepatol 46:1133-1142

56. Saadeh S, Younossi ZM, Remer EM, Gramlich T, Ong JP, Hurley M, Mullen KD, Cooper JN, Sheridan MJ (2002) The utility of radiological imaging in nonalcoholic fatty liver disease. Gastroenterology 123:745-750

57. Samuel VT, Liu ZX, Qu X, Elder BD, Bilz S, Befroy D, Romanelli AJ, Shulman GI (2004) Mechanism of hepatic insulin resistance in non-alcoholic fatty liver disease. J Biol Chem 279:32345-32353

58. Schwimmer JB, Behling C, Newbury R, Deutsch R, Nievergelt C, Schork NJ, Lavine JE (2005) Histopathology of pediatric nonalcoholic fatty liver disease. Hepatology 42:641-649

59. Schwimmer JB, Deutsch R, Kahen T, Lavine JE, Stanley C, Behling C (2006) Prevalence of fatty liver in children and adolescents. Pediatrics 118:1388-1393

60. Schwimmer JB, Middleton MS, Deutsch R, Lavine JE (2005) A phase 2 clinical trial of metformin as a treatment for non-diabetic paediatric non-alcoholic steatohepatitis. Aliment Pharmacol Ther 21: 871-879

61. Schwimmer JB, Pardee PE, Lavine JE, Blumkin AK, Cook S (2008) Cardiovascular risk factors and the metabolic syndrome in pediatric nonalcoholic fatty liver disease. Circulation 118:277-283

62. Shannon A, Alkhouri N, Carter-Kent C, Monti L, Devito R, Lopez R, Feldstein AE, Nobili V (2011) Ultrasonographic quantitative estimation of hepatic steatosis in children With NAFLD. J Pediatr Gastroenterol Nutr 53:190-195

63. Swiderska-Syn M, Suzuki A, Guy CD, Schwimmer JB, Abdelmalek MF, Lavine JE, Diehl AM (2013) Hedgehog pathway and pediatric nonalcoholic fatty liver disease. Hepatology 57: $1814-1825$

64. Tiniakos DG, Vos MB, Brunt EM (2010) Nonalcoholic fatty liver disease: pathology and pathogenesis. Annu Rev Pathol 5:145-171

65. Vajro P, Franzese A, Valerio G, Iannucci MP, Aragione N (2000) Lack of efficacy of ursodeoxycholic acid for the treatment of liver abnormalities in obese children. J Pediatr 136:739-743

66. Vajro P, Lenta S, Socha P, Dhawan A, McKiernan P, Baumann U, Durmaz O, Lacaille F, McLin V, Nobili V (2012) Diagnosis of nonalcoholic fatty liver disease in children and adolescents: position paper of the ESPGHAN Hepatology Committee. J Pediatr Gastroenterol Nutr 54:700-713

67. Vajro P, Mandato C, Franzese A, Ciccimarra E, Lucariello S, Savoia M, Capuano G, Migliaro F (2004) Vitamin E treatment in pediatric obesity-related liver disease: a randomized study. J Pediatr Gastroenterol Nutr 38:48-55

68. Vajro P, Mandato C, Licenziati MR, Franzese A, Vitale DF, Lenta S, Caropreso M, Vallone G, Meli R (2011) Effects of Lactobacillus rhamnosus strain GG in pediatric obesity-related liver disease. J Pediatr Gastroenterol Nutr 52:740-743

69. Wang CL, Liang L, Fu JF, Zou CC, Hong F, Xue JZ, Lu JR, Wu XM (2008) Effect of lifestyle intervention on non-alcoholic fatty liver disease in Chinese obese children. World J Gastroenterol 14:15981602

70. Weiner RA (2010) Surgical treatment of non-alcoholic steatohepatitis and non-alcoholic fatty liver disease. Dig Dis 28:274-279

71. Widhalm K, Ghods E (2010) Nonalcoholic fatty liver disease: a challenge for pediatricians. Int J Obes (Lond) 34:1451-1467

72. Xanthakos S, Miles L, Bucuvalas J, Daniels S, Garcia V, Inge T (2006) Histologic spectrum of nonalcoholic fatty liver disease in morbidly obese adolescents. Clin Gastroenterol Hepatol 4:226-232

73. Zelman S (1952) The liver in obesity. AMA Arch Intern Med 90: $141-156$ 